\title{
North American Nurses' and Doulas' Views of Each Other
}

Journal of Obstetric Gynecologic, \& Neonatal Nursing (JOGNN) 45(6): 790-800.

DOI: http://dx.doi.org/10.1016/j.jogn.2016.06.011

\author{
Louise Marie Roth \\ University of Arizona \\ Megan M. Henley \\ University of Colorado-Mesa \\ Marla Seacrist \\ California State University-Stanislaus \\ Christine H. Morton \\ Stanford University
}




\title{
North American Nurses' and Doulas’ Views of Each Other
}

\author{
Abstract \\ Objective: To analyze factors that lead nurses and doulas to have positive views of each \\ other.
}

Design: A multivariate analysis of a cross-sectional survey, the Maternity Support Survey.

Setting: $\quad$ Online survey with labor and delivery (L\&D) nurses, doulas, and childbirth educators, in the United States and Canada.

Participants: A convenience sample of 704 L\&D nurses and 1,470 doulas.

Methods: Multiple regression analysis was used to examine five sets of hypotheses about

10
nurses’ and doulas’ attitudes toward each other. Scales of nurses’ attitudes toward doulas and doulas’ attitudes toward nurses included beliefs that nurses/doulas enhance communication, are collaborative team members, enhance a woman’s birth experience, interfere with the ability to provide care, or interfere with relationships with patients/clients.

\section{Results:}

For nurses, exposure to doulas in their primary hospital was associated with more positive views, while working more hours, feelings of overwork, and a preference for clinical tasks over labor support were associated with more negative views of doulas. For doulas, working primarily in one hospital and certification were associated with more positive views of nurses. Nurses with more positive attitudes toward common obstetric practices had more negative attitudes toward doulas, while doulas with more positive attitudes toward common obstetric practices had more positive attitudes toward nurses. 
23 Conclusion: These findings reveal factors that influence mutual understanding and appreciation of nurses and doulas of each other. These factors can be influenced by educational efforts to improve interprofessional collaboration between these maternity support roles.

27 Key words: Inter-professional collaboration; Nurse-doula relationships; Maternity care;

Attitudes

\section{Call outs:}

31 1. Understanding labor nurse and doula attitudes toward each other may help to increase teamwork and improve maternity outcomes.

2. The finding that labor nurses and doulas do not share the same attitudes toward one

37 Précis: Differing attitudes toward obstetric procedures influence nurses’ and doulas’ views of

38 each other in the provision of labor support. More education and clarification of doulas' role is 39 needed to enhance collaborative practice. 
Labor support is an important source of emotional and physical comfort for women during childbirth that leads to shorter labors, fewer cesarean deliveries, increased breastfeeding, and higher maternal satisfaction (Barrett \& Stark, 2010; Flamm, Berwick, \& Kabcenell, 1998;

Gordon et al., 1999; Hodnett, Gates, Hofmeyr, \& Sakala, 2012; McGrath \& Kennell, 2008). Labor support includes emotional and physical support, information, advice, and advocacy (Bianchi \& Adams, 2004; Deitrick \& Draves, 2008; Rosen, 2004). In the contemporary U.S. and Canada, a family member or friend may act as a support person. Labor and delivery (L\&D) nurses or doulas can also provide labor support in occupational roles that we have called “maternity support workers” (MSWs). Multiple sources of labor support can complement each other, making collaboration in labor support more than the sum of its parts (Deitrick \& Draves, 2008; Morton, Seacrist, Torres, \& Heidbreder, 2015 in press; Rosen, 2004). Understanding those factors that influence labor nurse and doula attitudes toward each other can inform efforts to increase teamwork among these roles and possibly contribute to improved maternity outcomes.

Nearly 99\% of births in the U.S. and Canada take place in hospitals (Martin, Hamilton, Osterman, Curtin, \& Matthews, 2013; Statistics Canada, 2013). In a hospital setting, L\&D nurses are somewhat restricted in their ability to provide supportive care, because hospitals in the U.S. and Canada are not always able to provide for one-to-one nursing care during labor (Ballen \& Fulcher, 2006; Barrett \& Stark, 2010). In fact, hospitals may not follow professional nursing organizations' staffing guidelines, which outline when laboring patients should receive one-to- 
one care (Association of Women's Health Obstetric and Neonatal Nurses, 2010). As a result, teamwork with other MSWs like doulas can increase levels of support for women in labor. [Callout \#1 about here]

Partly in response to medicalized care and the increasing technological and documentation tasks required of L\&D nurses, doulas emerged as a specific labor support role over the last 35 years (Morton \& Clift, 2014). Doulas currently attend approximately 5-6\% of all U.S. births (Declercq et al., 2013). The percent of women who use doulas in Canada is unknown. Doulas provide continuous labor support to their clients and most doulas work in hospital settings, although most are not employed directly by hospitals or have a formal institutional role (Lantz et al., 2005). Doulas are not regulated or licensed, and there are no universally accepted standards for doula certification, academic preparation, training, or practice (Morton \& Clift, 2014). As a result, when doulas accompany their clients in the hospital, they carefully navigate and negotiate their role with maternity clinicians, including L\&D nurses with whom they share some overlapping roles and tasks (Henley, 2015; Torres, 2013). While doulas often work alongside nurses as they provide labor support, little is known about how nurses and doulas view each other's roles (Morton \& Clift, 2014).

\section{Theoretical Framework}

Several frameworks informed this study including social identity theory, theories about professional culture, and professional centrism. In social identity theory, personal identity combines with a group identity, where norms and attitudes of other members are shared (Tajfel, 
1981). Individual group members learn to see themselves through the lens of the group identity.

Social identity theorists focus on how the group is expressed within the individual rather than how individuals act within groups (Pecukonis, Doyle, \& Bliss, 2008). Like those in many occupations, maternity support workers often identify with their specific role as nurses or doulas, and its associated unique norms and attitudes.

We expand the notion of social identity and situate it within the construct of professional culture. Each health discipline possesses its own professional culture that determines core values, customs, symbols, meanings, and definitions of health, wellness, and treatment success (Pecukonis, 2014; Pecukonis et al., 2008). Professional culture also defines power distributions within the work environment, relationships between team members, and conflict resolution (Pecukonis et al., 2008). In maternity support work, professional cultures, in concert with social identity, shape the values and beliefs of labor nurses, and doulas.

Within the construct of professional culture is the concept of professional centrism. Similar to ethnocentrism, professional centrism is a preferred view of the world held by a particular occupational group; unfortunately, professional centrism leads to biased thinking that is based on stereotypes and prejudices (Pecukonis et al., 2008). When one professional group views their profession as more central or important than another group, this can negatively influence interdisciplinary cooperation.

\section{Background}

Doulas occupy a tenuous place in hospital-based birth due to their lack of integration in institutional settings (Norman \& Rothman, 2007; Torres, 2013). Most doulas are hired directly by, and primarily accountable to, pregnant women rather than institutions or their policies (Morton \& Clift, 2014). Some doulas, however, are more embedded in hospital settings than 
others. Doulas who work primarily at one hospital are likely to develop relationships with the L\&D nursing staff, which may improve the attitudes of nurses and doulas toward each other (the “exposure effect”) (Deitrick \& Draves, 2008; Grush, 1976; Zajonc, 1968). In psychology, the “exposure effect” refers to the idea that mere repeated exposure of individuals to the same stimulus will enhance their attitudes toward it (Akhavan \& Lundgren, 2012; Zajonc, 1968). It follows that L\&D nurses with repeated exposure to doulas would develop more positive attitudes toward them.

Doulas vary in their level of professionalization, with many doulas obtaining certification (Henley, 2015; Morton \& Clift, 2014; Roth et al., 2014). While not required, certification typically requires doulas to agree to practice within the parameters of their certifying organization, which may increase their legitimacy among maternity clinicians (Henley, 2015; Morton \& Clift, 2014). Certified doulas may also be more likely to respect boundaries between their role and that of nurses and may be more open to building positive relationships with clinicians (Henley, 2015).

Labor and delivery nurses are institutionally-embedded clinical practitioners who focus on and monitor the health and well-being of mothers and fetuses (Morton \& Clift, 2014). Nurses have multiple responsibilities: they must follow institutional policies and implement providers’ orders while caring for several patients (Morton et al., 2015 in press). As hospital employees, L\&D nurses often face constraints in providing labor support due to staffing patterns, documentation responsibilities, and/or barriers within the hospital culture (Barrett \& Stark, 2010; Gilliland, 2011; Rosen, 2004). L\&D nurses might react to these challenges by embracing doulas, although one study of a hospital-based doula program found that nurses appreciated the doula's 
presence, but they often did not fully understand the doula role or see it as distinct from the supportive role of friends or family (Deitrick \& Draves, 2008).

Educational strategies can improve L\&D nurses’ and doulas’ familiarity with each other’s scope of practice (Gilliland, 2002). Advice about improving L\&D nurse-doula collaboration includes a recommendation for nurses to explain the sequence of events to doulas, both at admission and again before any procedures (Bowers, 2002; Tumblin \& Simkin, 2001). However, this advice requires a substantial time commitment, and overworked nurses may find it difficult to follow. This situation is exacerbated by the fact that many doulas tend to be relatively inexperienced because the on-call doula lifestyle and the intense emotional nature of the work are difficult to sustain (Morton \& Clift, 2014).

\section{Attitudes}

An attitude is a settled way of thinking or feeling about someone or something. Most attitudes are socially learned, where group membership has a strong influence on attitudes (Hogg, 2006; Hogg, 2012). Doula training is heavily influenced by the midwifery model of care, which promotes normal (physiological) birth, and studies find that doulas tend to be critical of non-medically indicated interventions in childbirth (Basile, 2012; Morton \& Clift, 2014; Norman \& Rothman, 2007; Stevens, Dahlen, Peters, \& Jackson, 2011). In contrast, most nurses have training and experience in standard hospital-based models of obstetric care. There is some evidence that nurses with more experience hold more positive attitudes toward labor support and more negative views of technology and interventions than less experienced nurses (Carlton, Callister, Christiaens, \& Walker, 2009; James, Simpson, \& Knox, 2003; Sleutel, Schultz, \& Wyble, 2007). However, Liva, Hall, Klein and Wong (2012) found weak or no relationship between L\&D nurses’ years of experience and attitudes toward birth practices (Liva et al., 2012). 


\section{Hypotheses}

Based on the literature review and theoretical framework, we tested five main hypotheses about L\&D nurses’ and doulas' views of each other. First, doulas who work primarily in one hospital, earn more from doula work, and/or are certified will have more positive views of nurses $\left(\mathrm{H}_{1}\right)$. Second, nurses working in hospitals where doulas attend a higher percentage of births and those with more years of experience will have more positive views of doulas $\left(\mathrm{H}_{2}\right)$. Third, nurses who work more hours, attend more births per shift, and feel overworked will have more negative views of doulas $\left(\mathrm{H}_{3}\right)$. Fourth, nurses who value labor support as much as, or more than, clinical tasks involved in observation and treatment of patients will have more positive views of doulas $\left(\mathrm{H}_{4}\right)$. Finally, nurses with more positive attitudes toward typical obstetric protocols will have more negative views of doulas, while doulas with more positive attitudes toward typical obstetric protocols will have more positive views of nurses $\left(\mathrm{H}_{5}\right)$.

\section{Methods}

Data for this study come from the Maternity Support Survey (MSS), a cross-sectional online survey of labor and delivery (L\&D) nurses, doulas, and childbirth educators in the United States and Canada. The survey recruited participants between November 2012 and March 2013 through each occupation's professional associations. The organizations for nurses were the Association of Women's Health, Obstetric and Neonatal Nurses (AWHONN) and the Canadian Nurses Association (CNA). The doula organizations were DONA International, Health Connect One, toLABOR (formerly ALACE), CAPPA Canada, and Doula C.A.R.E. (Canada). We also recruited childbirth educators through Lamaze International, International Childbirth Education Association (ICEA), Birthing from Within, and BirthWorks. The professional organizations emailed their current members a recruitment letter via email with a link to the survey, followed 
by up to two reminders. The research team also publicized the survey to other labor and delivery nurses, doulas, and childbirth educators via social media (Facebook, Twitter, and maternity blogs) and email networks. The survey collected no personal identifiers, and the Institutional Review Board at the University of Arizona determined the study to be exempt. A total of 3,325 respondents started the survey and 2,781 completed it, for a completion rate of $83.6 \%$. For this analysis, we excluded respondents who worked exclusively as childbirth educators, because they were unlikely to provide active labor support.

The survey consisted of questions about demographic characteristics, training and credentials in the maternity support field, sources of information and knowledge about birth, financial rewards of maternity support work, childbirth and breastfeeding experience, attitudes toward common labor practices and breastfeeding, attitudes toward other maternity support roles, work experiences including ethical challenges, work satisfaction and burnout, hospital characteristics, understandings of informed consent, experiences with and knowledge of quality improvement initiatives, and questions specific to each maternity support role (Roth et al., 2014).

Demographic characteristics included age (in years), level of education, race-ethnicity, household income, and marital status (married=1). (We also coded partnership status as partnered/unpartnered by aggregating married individuals and those in long-term domestic partnerships, and this variable produced the same results.) Over 99.5\% of respondents identified as female. We examine regional effects for Canada and the Northeastern, Midwestern, Southern, and Western Census regions in the United States

(http://www.census.gov/geo/www/us_regdiv.pdf). The multivariate models omit the indicator for West as the reference category. (One must always include one less indicator variable in the 
model than there are categories, in order to avoid introducing multicollinearity into the model. All other regional coefficients represent the regions’ difference with West.)

Features of respondents' maternity support position included their income from their maternity support job. Because more than half of doulas earned less than $\$ 5,000$ from doula work and very few earned $\$ 40,000$ or more (1.2\% of doulas), we aggregated doula income into 7 categories, with a top code of $\$ 40,000$ or more. Nurse incomes are measured in 12 categories, from $1=<\$ 5,000$ to $12=\$ 100,000$ or more. For doulas, this study includes indicators for certification and whether they work primarily at one hospital. Measures for nurses include percent of births at their primary hospital that have doula support, years of nursing experience, hours per week, estimated average number of births per 10 hours of work, feelings of overwork (an indicator for working more than one would prefer), and whether they enjoy labor support as much as or more than clinical tasks (e.g., monitoring vital signs, administering medication).

The survey measured attitudes with 5-point Likert scales from 1 (strongly disagree) to 5 (strongly agree). We constructed standardized scales with ranges of 0-10, ranked from extremely negative to extremely positive, for attitudes toward epidural analgesia, induction, and cesarean delivery. Table 1 enumerates the items in each scale. The scales with the weakest internal reliability were those for attitudes toward induction and cesarean delivery. However, following Liva et al. (2012), we retained these scales because a Cronbach's alpha below 0.7 is common for attitudinal scales and attitudes about standard obstetric practices are theoretically relevant for attitudes about nurse-doula relationships (Kline, 2000). The remaining scales for attitudes toward epidural analgesia and the dependent variables had moderate to strong internal reliability.

[Table 1 about here] 
Two scales measuring nurses’ attitudes toward doulas and doulas’ attitudes toward nurses are the dependent variables for this study (see Table 2). Each of these scales aggregates five attitudes, measured from 1 to 5 . We subtracted 5 from the scales to standardize the range from 0 20, with a value of 0 representing a strongly negative view, and a value of 20 representing a strongly positive view. The internal consistency reliability of both scales was strong ( $\alpha=0.91$ for nurses' attitudes toward doulas and $\alpha=0.86$ for doula attitudes toward nurses). As a robustness check, we conducted a principal components factor analysis, which is a method of data reduction that seeks underlying, unobservable (latent) variables that the observed variables reflect. For both scales, this extracted a single factor that explained a substantial amount of the variance in the 5 independent measures and provided additional evidence that the 5 measures capture a single underlying construct.

Data Analysis

We treated scores on nurses’ attitudes toward doulas and doulas’ attitudes toward nurses as continuous data for this analysis and we conducted Ordinary Least Squares (OLS) multiple regression analysis with robust standard errors. OLS regression is a generalized linear modelling technique that models the linear effects of predictor variables on an interval-level response variable, like the 0-20 attitudinal scales. While our models exhibited very strong conformity with OLS assumptions, robust standard errors adjust for a collection of minor concerns about failure to meet OLS assumptions and are considered a best practice in statistical modeling. (Plots of the unadjusted residuals demonstrated that the models conformed very well to OLS assumptions of uncorrelated and normally-distributed error terms.) The models examine the linear relationship between independent variables (predictors) and attitudes toward nurse-doula collaboration. (Ordinal logistic models that treated the dependent variables as rank-ordered variables had nearly 
identical results.) Predictors included demographic controls (age, education, race, household income, marital status), region (West=reference), and the scales of attitudes toward common obstetric practices of epidural analgesia, induction, and cesarean delivery (see Table 1). For the model of doulas' attitudes toward nurses, we included certification status, income from doula work, and an indicator for working primarily in one hospital as measures of doulas' institutional embeddedness. For the model of nurses’ attitudes toward doulas, we included percent of births with a doula in attendance at their primary hospital, years of nursing experience, income from nursing, hours worked per week, average number of births per 10 hours of work, and an indicator for feeling overworked (working more hours than one prefers). In order to include the measure of doula exposure, we had to exclude from the model those nurses who did not work primarily at one hospital. We coded nurses who indicated that they enjoyed labor support as much or more than clinical tasks as enjoying labor support.

Although the total sample included 1,569 doulas and 1,012 nurses, there were 161 cases with missing values on relevant variables (99 doulas and 62 nurses), representing $6.2 \%$ of cases. We used listwise deletion for missing data after we compared missing to non-missing cases: there were no significant differences in the dependent variables, and a few very small differences in the independent variables. We also excluded 246 nurses who did not work primarily at one hospital. Thus, the analyses include 1,470 doulas and 704 nurses. Using a power analysis for multiple regression, we determined the minimum sample sizes to estimate a medium effect size with an alpha of 0.05 using 18 predictor variables (doula model) or 20 predictor variables (nurse model), to be 149 and 156 respectively (http://www.danielsoper.com/statcalc3/calc.aspx?id=1). The sample sizes for the models are significantly larger than these minimum sizes, and have $>99 \%$ power to reject the null hypothesis if a relationship is present. 


\section{Results}

Table 2 presents descriptive statistics and metrics for the dependent variables and all predictors in the regression models. The average age of participants was 47 for L\&D nurses, and 40 for doulas. The vast majority of respondents were white. Nurses had more education and higher household incomes than doulas, on average. Nurses had an average of 21.5 years of experience, worked an average of 31 hours per week, and attended an average of 1 birth per 10 hours of work. The vast majority (93\%) of nurses indicated that they enjoyed labor support at least as much as clinical tasks. Among doulas, a majority was certified (69\%) and about one quarter (26\%) worked primarily in one hospital. Doulas’ average income from doula work was less than $\$ 9,999$, while nurses’ average income from nursing was $\$ 60,000-\$ 69,999$. In general, nurses were slightly more positive toward induction than doulas, considerably more positive toward epidural analgesia, and somewhat more positive toward cesarean deliveries (although both groups tended to have negative views of cesarean delivery).

[Table 2 about here]

Table 3 presents the results of OLS regression models for nurses’ attitudes toward doulas and doulas’ attitudes toward nurses. Most demographic characteristics had no significant impact on these MSWs' views, except that higher education and household income are associated with more positive views of nurses for doulas $(\mathrm{p}<0.1)$. In contrast, nurses with higher household incomes have more negative views of doulas. Older nurses appear to have more positive views of doulas, controlling for their years of nursing experience (While age and years in nursing were correlated, this did not appear to present problems for the models because of the large sample size, so we retained both variables in the models).

[Table 3 about here] 
For doulas, working primarily at one hospital and being certified both have a significant positive effect on their views of nurses, but income from doula work does not. This partially supports the hypothesis $\left(\mathrm{H}_{1}\right)$ that more institutionally embedded doulas will have more positive relationships with nurses. The results also largely support the hypothesis $\left(\mathrm{H}_{5}\right)$ that doulas with more positive attitudes toward common obstetric practices will have more positive views of L\&D nurses, specifically with respect to epidural analgesia and induction. There are also regional effects, with doulas in the northeastern or southern United States or in Canada having significantly more negative views of nurses than doulas in the Western United States. Doulas may be more common and more institutionally embedded in some regions, leading to more exposure of nurses to doulas. Overall, the variables in this model explain approximately $8 \%$ of the variance in doulas' views of their relationships with nurses, as indicated by the $\mathrm{R}^{2}$ statistic.

The model of nurses’ views of doulas in Table 3 offers a stronger explanatory model, explaining approximately $27 \%$ of the variance. This partially supports $\mathrm{H}_{2}$, which hypothesized that more exposure to doulas would be associated with more positive views, although it fails to support the prediction $\left(\mathrm{H}_{2}\right)$ that more years of nursing experience will be associated with more positive views of doulas. There is also support in this model for the hypothesis $\left(\mathrm{H}_{3}\right)$ that overworked nurses are less favorable toward doulas, since the number of hours that nurses work per week and working more than they want to work both have significant negative effects. However, the number of births per 10 hours of nursing work did not have a significant effect on nurses’ views of doulas. Supporting $\mathrm{H}_{4}$, nurses who enjoy labor support as much as, or more than, clinical tasks have a more positive view of doulas. Since 93\% of nurses indicated that they enjoyed labor support at least as much as clinical tasks, this may say more about nurses who prefer clinical tasks having negative views of doulas than it does about the vast majority who 
enjoy labor support. In support of hypothesis $\left(\mathrm{H}_{5}\right)$, we observe the opposite effect of attitudes toward common labor practices for nurses as for doulas: all else being equal, nurses with more positive views of epidural analgesia, induction, and cesarean deliveries are significantly more negative about doulas.

\section{Discussion}

Household income, controlling for maternity support income, is a measure of socioeconomic status (SES) and it is possible that higher-SES doulas know more nurses in their non-professional social networks or view them as socioeconomic equals. The opposite may be the case for nurses: higher-SES nurses may know fewer doulas outside of their professional life.

Team practices among health care professionals require mastery of certain skills, knowledge, and attitudes (Interprofessional Education Collaborative, 2011). Collaboration is both a skill and an attitude. Our study revealed that nurses who work with doulas more often and nurses who value labor support have more favorable views of doulas. Additionally, doulas who attend more births and who are certified have more positive attitudes toward nurses. Viewed through the lens of interprofessional cultural competence, these findings demonstrate that mutual understanding and appreciation of the roles and contributions of each discipline may be a factor in achieving effective interdisciplinary practice (Pecukonis et al., 2008). Collaboration also improves team decision-making, planning, and goal setting (Lyndon et al., 2015; Pecukonis et al., 2008), and these findings suggest that nurse-doula collaboration may be one implementation strategy in efforts to improve patient outcomes in the childbirth setting.

However, the single most intractable barrier to interprofessional education and collaboration is professional centrism or professional culture (Pecukonis, 2014). The finding that labor nurses and doulas do not share the same attitudes toward one another indicates that some 
“turf” issues still exist (Pecukonis, 2014). While each occupation has its own culture, collaboration promotes interdisciplinary practice, which in turn reduces professional centrism (Pecukonis et al., 2008). Since certified doulas had more favorable attitudes toward nurses, it is likely that their certification process provided interdisciplinary education and encouraged collaborative values. In contrast, nurses who were overworked (more births) had more negative attitudes toward doulas. Although we suspect that the negativity might come from having too little time to spend with less experienced doulas, overworked nurses might also perceive a doula's presence as a disruption in the routine care they would otherwise provide. While it may seem counter-intuitive that overworked nurses would not perceive doulas' labor support activities as helping to reduce their workload, it is possible that these nurses view doulas as one more maternity team member to direct, collaborate with, and get reports from, thus adding to their work.

\section{[Callout \#2 about here]}

Of interest were the contrasting effects of attitudes toward typical obstetric practices on nurses' and doulas' views of each other. Doulas who had a more favorable attitude toward Pitocin and epidurals had more positive attitudes toward nurses, suggesting greater overall comfort with typical labor management in hospitals. In contrast, nurses who expressed favorable attitude toward obstetric procedures showed more negative attitudes toward doulas, possibly viewing them as impediments to smooth protocol implementation. These results suggest that the differing attitudes toward obstetric procedures in nurses’ and doulas’ professional cultures constrain doula-nurse collaboration, and that collaboration is more likely when these groups understand each other's professional culture. In addition, nurse-doula collaboration is likely to enhance a woman’s birth experience (Papagni \& Buckner, 2006). 
From our review of the literature, as well as our clinical expertise, we believe the following practices could enhance nurse-doula relationships in the hospital setting:

- Mutual introductions that take place inside or outside the patient room.

- Commit to mutual respect for one another, supporting the unique role each has to offer

- Representative trainer/educators from both roles can participate in each other's workshops/classes, with nurses teaching a doula workshop segment on hospital roles and routines and doulas teaching labor support to nurses.

- New labor nurses and doulas could spend time shadowing their counterparts through an entire labor and birth, to learn the other's role and inform their own support techniques.

- Joint educational conferences could be held between doula and nursing organizations.

\section{Limitations}

The Maternity Support Survey (MSS) is the first cross-national survey to examine the under-studied roles and views of labor nurses and doulas in the United States and Canada. The survey obtained a large number of responses from MSWs. A limitation of the study is that recruitment methods resulted in a non-random sample, so responses may not be generalizable to all L\&D nurses and doulas in the U.S. and Canada. This sampling method also precluded our ability to calculate a response rate, since we did not have a denominator. More specifically, most respondents were members of professional organizations that assisted with the study, but many MSWs, especially L\&D nurses, are not members of these specific organizations and thus were reached through social media recruitment methods described above.

\section{Conclusion}

[Callout \#3 about here] 
The results from the Maternity Support Survey (MSS) reveal factors that influence positive attitudes of nurses and doulas toward each other. With national efforts underway to address worsening maternity outcomes in both countries, particularly high primary cesarean rates, interprofessional communication and collaboration are essential to enhance patient safety and satisfaction (Lyndon et al., 2015). A collegial and collaborative nurse-doula relationship is one possible strategy for hospitals to consider in their efforts to reduce primary cesareans. Even when nurses desire and know how to provide hands-on labor support, they are often unable to do so due to workplace constraints, such as documentation tasks, ineffective staffing patterns, institutional policies, and professional centrism (Barrett \& Stark, 2010; Gilliland, 2002; Gilliland, 2011; Rosen, 2004). Nurses and doulas working together can ensure that laboring women have their desired levels of non-pharmacological pain relief and continuous support. Women who have continuous labor support report being more satisfied with their births. In addition, doulas are one of the most effective components among efforts to provide continuous labor support, which in turn has been shown to reduce cesarean births (American Congress of Obstetricians and Gynecologists, 2014; Berghella, Baxter, \& Chauhan, 2008). The results of this study point to factors that affect whether nurses will have positive views of doulas. Our findings reveal that not all L\&D nurses enjoy labor support - unit managers may utilize this information in their assignments or in when they design continuing education curricula. Professional associations can educate and train their members on the value of nurse-doula collaboration, while individual nurses and doulas can model collaborative behavior in each encounter with one another. Optimizing maternal-infant outcomes is a shared goal of both nurses and doulas, and improved collaboration among all MSWs will help achieve this goal. 
Table 1: Attitude Scales

\begin{tabular}{|l|l|l|}
\hline Scale & Subscale Items (1=Strongly disagree, to 5=Strongly agree) & $\alpha$ \\
\hline $\begin{array}{l}\text { Epidural } \\
\text { analgesia }\end{array}$ & $\begin{array}{l}\text { Epidurals should be routinely offered to all women in labor } \\
\text { Epidurals conserve maternal energy for the second stage of labor } \\
\text { Epidurals interfere with the normal progress of labor (reverse coded) } \\
\text { Epidurals increase the risk of cesarean delivery (reverse coded) }\end{array}$ & 0.80 \\
\hline Induction & $\begin{array}{l}\text { Induction is safe as part of a standardized protocol } \\
\text { Induction increases the need for epidural analgesia (reverse coded) } \\
\text { Inductions increase the cesarean rate (reverse coded) }\end{array}$ & 0.67 \\
\hline $\begin{array}{l}\text { Cesarean } \\
\text { Delivery }\end{array}$ & $\begin{array}{l}\text { Cesareans prevent urinary incontinence } \\
\text { Cesareans are safer for babies than vaginal births } \\
\text { Vaginal birth often compromises a woman's sexual functioning after birth } \\
\text { Cesareans are as safe for women as vaginal births } \\
\text { Women should be able to choose CS, even in absence of medical indication } \\
\text { For a woman with a previous CS, scheduled repeat CS is the best for her health } \\
\text { For a woman with a previous CS, scheduled repeat CS reduces litigation risk } \\
\text { A woman who is a good candidate for VBAC should be able to attempt it if she } \\
\text { desires }\end{array}$ & 0.71 \\
\hline $\begin{array}{l}\text { Durses enhance communication around childbirth procedures } \\
\text { Views of } \\
\text { Nurses }\end{array}$ & $\begin{array}{l}\text { Nurses are a collaborative team member } \\
\text { Nurses enhance the woman's birth experience } \\
\text { Nurses interfere with my ability to provide care (reverse coded) } \\
\text { Nurses interfere with the doula-client relationship (reverse coded) }\end{array}$ & \\
\hline $\begin{array}{l}\text { Doulas enhance communication around childbirth procedures } \\
\text { Doulas are a collaborative team member } \\
\text { Doulas enhance the woman's birth experience } \\
\text { Doulas interfere with my ability to provide care (reverse coded) } \\
\text { Doulas interfere with the nurse-patient relationship (reverse coded) }\end{array}$ & 0.86 \\
\hline $\begin{array}{l}\text { Views of } \\
\text { Doulas }\end{array}$ & \\
\hline
\end{tabular}


Table 2: Descriptive Statistics for Nurses and Doulas in the Maternity Support Survey

\begin{tabular}{|c|c|c|c|}
\hline VARIABLE & METRIC & $\begin{array}{c}\text { DOULAS } \\
\text { Mean (SD) or \% }\end{array}$ & $\begin{array}{l}\text { L\&D NURSES } \\
\text { Mean (SD) or \% }\end{array}$ \\
\hline View of Doulas/Nurses & Scale $0-20$ & $12.95(3.15)$ & $11.89(4.32)$ \\
\hline \multicolumn{4}{|l|}{ Demographics } \\
\hline Age & In years & $40.44(11.76)$ & 47.08 (10.98) \\
\hline Education & $1=\mathrm{HS}$ or less to $5=$ Doctorate & $2.76(0.85)$ & $2.99(0.72)$ \\
\hline White & $1=$ yes & $93 \%$ & $95 \%$ \\
\hline $\mathrm{HH}$ income & $1=<\$ 20 \mathrm{k}$ to $7=\$ 150 \mathrm{k}+$ & $4.10(1.66)$ & $5.38(1.19)$ \\
\hline Married & $1=$ yes & $69 \%$ & $72 \%$ \\
\hline \multicolumn{4}{|l|}{ Region } \\
\hline Northeast & $1=$ yes & $17 \%$ & $17 \%$ \\
\hline Midwest & $1=$ yes & $19 \%$ & $23 \%$ \\
\hline South & $1=$ yes & $19 \%$ & $26 \%$ \\
\hline West (reference) & $1=$ yes & $26 \%$ & $22 \%$ \\
\hline Canada & $1=$ yes & $15 \%$ & $8 \%$ \\
\hline \multicolumn{4}{|c|}{ Features of Maternity Support Position } \\
\hline One hospital & $1=$ yes & $26 \%$ & - \\
\hline Doula income & $0=$ volunteer to $6=\$ 40 \mathrm{k}+$ & $1.35(1.15)$ & - \\
\hline Doula certification & $1=$ yes & $69 \%$ & - \\
\hline \% Births with a Doula & & - & $6.26(8.14)$ \\
\hline Nursing experience & In years & - & $21.47(11.58)$ \\
\hline Nursing income & $1=<\$ 20 \mathrm{k}$ to $10=\$ 100 \mathrm{k}+$ & - & $8.19(2.40)$ \\
\hline Nursing hours per week & In hours & - & $31.20(14.54)$ \\
\hline Births per 10 hours & $\begin{array}{l}\text { Estimated \# births per } 10 \\
\text { hours of work }\end{array}$ & - & $1.00(0.99)$ \\
\hline Works more than desired & $1=$ yes & - & $0.26(0.44)$ \\
\hline Enjoys labor support & $1=$ yes & - & $93 \%$ \\
\hline \multicolumn{4}{|l|}{ Attitude Scales } \\
\hline Positive about epidurals & Scale $0-10$ & $2.40(1.44)$ & $5.42(2.04)$ \\
\hline Positive about induction & Scale $0-10$ & $1.67(1.33)$ & $3.56(1.73)$ \\
\hline Positive about cesareans & Scale $0-10$ & $1.56(1.03)$ & $2.53(1.33)$ \\
\hline $\mathrm{N}$ & & 1,470 & 704 \\
\hline
\end{tabular}


Table 3: Regression Analysis Predicting Attitudes toward Doula-Nurse Relationships (on a scale of 0-20)

DOULAS (Robust SE) NURSES (Robust SE)

Demographics

\begin{tabular}{|c|c|c|c|c|}
\hline Age & 0.004 & $(0.01)$ & $0.05 \dagger$ & $(0.02)$ \\
\hline Education & $0.16 \dagger$ & $(0.09)$ & 0.12 & $(0.19)$ \\
\hline White & -0.02 & $(0.30)$ & 0.59 & $(0.68)$ \\
\hline HH income & $0.10 \dagger$ & $(0.05)$ & $-0.31^{*}$ & $(0.14)$ \\
\hline Married & -0.20 & $(0.21)$ & 0.14 & $(0.37)$ \\
\hline \multicolumn{5}{|l|}{ Region } \\
\hline Northeast & $-0.58 *$ & $(0.24)$ & -0.39 & $(0.45)$ \\
\hline Midwest & 0.17 & $(0.23)$ & $0.84^{*}$ & $(0.42)$ \\
\hline South & $-0.56^{*}$ & $(0.23)$ & 0.00 & $(0.44)$ \\
\hline Canada & $-0.80 * *$ & $(0.30)$ & -0.85 & $(0.67)$ \\
\hline \multicolumn{5}{|l|}{ Maternity Support Position } \\
\hline One hospital & $0.56^{* *}$ & $(0.19)$ & & \\
\hline Doula income & -0.07 & $(0.07)$ & & \\
\hline Doula certification & $0.40^{*}$ & $(0.18)$ & & \\
\hline \% Births with a Doula & \multicolumn{2}{|c|}{--} & $0.10 * * *$ & $(0.02)$ \\
\hline Nursing experience & \multicolumn{2}{|c|}{--} & -0.03 & $(0.02)$ \\
\hline Nursing income & \multicolumn{2}{|c|}{--} & -0.02 & $(0.08)$ \\
\hline Nursing hours per week & \multicolumn{2}{|c|}{--} & $-0.02 \dagger$ & $(0.01)$ \\
\hline Births per 10 hours & \multicolumn{2}{|c|}{--} & -0.22 & $(0.16)$ \\
\hline Works more than desired & \multicolumn{2}{|c|}{--} & $-0.62 \dagger$ & $(0.35)$ \\
\hline Enjoys labor support & \multicolumn{2}{|c|}{--} & $1.09 \dagger$ & $(0.57)$ \\
\hline \multicolumn{5}{|l|}{ Attitude Scales } \\
\hline Positive about epidurals & \multicolumn{2}{|l|}{$0.34 * * *$} & \multicolumn{2}{|l|}{$-0.47^{* * *}$} \\
\hline Positive about induction & \multicolumn{2}{|l|}{$0.24 * * *$} & $-0.21 *$ & $(0.10)$ \\
\hline Positive about cesareans & \multicolumn{2}{|l|}{$-0.05 * * *$} & $-0.54 * * *$ & $(0.12)$ \\
\hline Constant & \multicolumn{2}{|l|}{$10.92 * * *$} & $15.26 * * *$ & $(1.65)$ \\
\hline $\mathrm{R}^{2}$ & \multicolumn{2}{|c|}{$0.08 * * *$} & \multicolumn{2}{|c|}{$0.25 * * *$} \\
\hline $\mathrm{N}$ & \multicolumn{2}{|c|}{1,470} & \multicolumn{2}{|c|}{704} \\
\hline
\end{tabular}




\section{References}

American Congress of Obstetricians and Gynecologists. (2014). Safe prevention of the primary cesarean delivery. Obstetric care consensus practice bulletin. Retrieved from http://www.acog.org/Resources-And-Publications/Obstetric-Care-Consensus-Series/SafePrevention-of-the-Primary-Cesarean-Delivery

Akhavan, S., \& Lundgren, I. (2012). Midwives' experiences of doula support for immigrant women in Sweden: A qualitative study. Midwifery, 28(1), 80-85.

Association of Women's Health Obstetric and Neonatal Nurses. (2010). Guidelines for professional registered nurse staffing for perinatal units. AWHONN. Washington, D.C.

Ballen, L. E., \& Fulcher, A. J. (2006). Nurses and doulas: Complementary roles to provide optimal maternity care. Journal of Obstetric, Gynecologic, and Neonatal Nursing, 35(2), 304-311.

Barrett, S. J., \& Stark, M. A. (2010). Factors associated with labor support behaviors of nurses. Journal of Perinatal Education, 19(1), 12-18. doi: 10.1624/105812410X481528

Basile, M. (2012). Reproductive justice and childbirth reform: Doulas as agents of social change. PhD (Doctor of Philosophy) thesis, University of Iowa, 2012. Retrieved from http://ir.uiowa.edu/etd/2819

Berghella, V., Baxter, J. K., \& Chauhan, S. P. (2008). Evidence-based labor and delivery management. American Journal of Obstetrics \& Gynecology, 199(5), 445-454. doi: 10.1016/j.ajog.2008.06.093

Bianchi, A. L., \& Adams, E. D. (2004). Doulas, labor support, and nurses. International Journal of Childbirth Education, 19(4), 24-30. 
Bowers, B. B. (2002). Mothers' experiences of labor support: Exploration of qualitative research. Journal of Obstetric, Gynecologic and Neonatal Nursing, 31(6), 742-752.

Carlton, T., Callister, L. C., Christiaens, G., \& Walker, D. (2009). Labor and delivery nurses' perceptions of caring for childbearing women in nurse-managed birthing units. MCN: American Journal of Maternal Child Nursing, 34(1), 50-56. doi:

10.1097/01.NMC.0000343866.95108.fa

Declercq, E. R., Sakala, C., Corry, M. P., Applebaum, S., \& Herrlich, A. (2013). Listening to Mothers III: Pregnancy and Birth. New York: Childbirth Connection.

Deitrick, L., \& Draves, P. R. (2008). Attitudes towards doula support during pregnancy by clients, doulas and labor-and-delivery nurses: A case study from Tampa, Florida. Human Organization, 67(4), 397-406.

Flamm, B. L., Berwick, D. M., \& Kabcenell, A. (1998). Reducing cesarean section rates safely: Lessons from a "Breakthrough Series" collaborative. Birth, 25(2), 117-124.

Gilliland, A. L. (2002). Beyond holding hands: The modern role of the professional doula. Journal of Obstetric, Gynecologic and Neonatal Nursing, 31(6), 762-769.

Gilliland, A. L. (2011). After praise and encouragement: Emotional support strategies used by birth doulas in the USA and Canada. Midwifery, 27, 525-531.

Gordon, N. P., Walton, D., McAdam, E., Derman, J., Gallitero, G., \& Garrett, L. (1999). Effects of providing hospital-based doulas in health maintenance organization hospitals. Obstetrics and Gynecology, 93(3), 422-426.

Grush, J. E. (1976). Attitude formation and mere exposure phenomena: A nonartifactual explanation of empirical findings. Journal of Personality and Social Psychology, 33, 281290. 
Henley, M. M. (2015). Alternative and authoritative knowledge: The role of certification for defining expertise among doulas. Social Currents, (2)3, 260-279.

Hodnett, E. D., Gates, S., Hofmeyr, G. J., \& Sakala, C. (2012). Continuous support for women during childbirth. Cochrane Database of Systematic Reviews, 10(Art. No.: CD003766). doi: DOI: 10.1002/14651858.CD003766.pub4

Hogg, M. A. (2006). Social identity theory. In P. J. Burke (Ed.), Contemporary social psychological theories (pp. 111-136). Stanford, CA: Stanford University Press.

Hogg, M. A. (2012). Social identity and the psychology of groups. In M. R. Leary \& J. P. Tangney (Eds.), Handbook of self and identity (pp. 502-519). New York, NY: Guilford Press.

Interprofessional Education Collaborative. (2011). Core competencies for interprofessional collaborative practice: Report of an expert panel. Washington, D.C. Interprofessional Education Collaborative.

James, D. C., Simpson, K. R., \& Knox, G. E. (2003). How do expert labor nurses view their role? Journal of Obstetric, Gynecologic and Neonatal Nursing, 32(6), 814-823.

Kline, P. (2000). The handbook of psychological testing. New York, NY: Routledge.

Lantz, P., Low, L.K., Varkey, S. and Watson, R.L. (2005). Doulas as childbirth paraprofessionals: Results from a national survey. Women's Health Issues, 15: 109-116. doi:10.1016/j.whi.2005.01.002

Liva, S. J., Hall, W. A., Klein, M. C., \& Wong, S. T. (2012). Factors associated with differences in Canadian perinatal nurses' attitudes toward birth practices. Journal of Obstetric, Gynecologic and Neonatal Nursing, 41(6), 761-773. doi: 10.1111/j.15526909.2012.01412.x 
Lyndon, A., Johnson, M. C., Bingham, D., Napolitano, P. G., Joseph, G., Maxfield, D. G., \& O'Keeffe, D. F. (2015). Transforming communication and safety culture in intrapartum care: A multi-organization blueprint. Journal of Midwifery and Women's Health. doi: 10.1111/jmwh.12235

Martin, J. A., Hamilton, B. E., Osterman, M. J., Curtin, S. C., \& Matthews, T. J. (2013). Births: Final data for 2012. National vital statistics reports: From the Centers for Disease Control and Prevention, National Center for Health Statistics, National Vital Statistics System, 62(9), 1-68.

McGrath, S. K., \& Kennell, J. H. (2008). A randomized controlled trial of continuous labor support for middle-class couples: Effect on cesarean delivery rates. Birth, 35(2), 92-97. doi: 10.1111/j.1523-536X.2008.00221.X

Morton, C. H., \& Clift, E. G. (2014). Birth ambassadors: Doulas and the re-emergence of woman-supported birth in America. Amarillo, TX: Praeclarus Press.

Morton, C. H., Seacrist, M., Torres, J., \& Heidbreder, N. (2015 in press). Cultivating collaborative relationships between doulas and labor and delivery nurses in the provision of labor support. In A. Castaneda \& J. Searcy (Eds.), Intimate care: Doulas and the birthing body. Bradford, Ontario: Demeter Press.

Norman, B. M., \& Rothman, B. K. (2007). The new arrival: Labor doulas and the fragmentation of midwifery and caregiving. In W. Simonds, B. K. Rothman, \& B. M. Norman (Eds.), Laboring on: Birth in transition in the United States. New York: Routledge.

Papagni, K., \& Buckner, E. (2006). Doula support and attitudes of intrapartum nurses: A qualitative study from the patient's perspective. The Journal of Perinatal Education, 15(1), 11-18. doi: 10.1624/105812406X92949 
Pecukonis, E. (2014). Interprofessional education: A theoretical orientation incorporating profession-centrism and social identity theory. Journal of Law and Medical Ethics, 42 Suppl 2, 60-64. doi: 10.1111/jlme.12189

Pecukonis, E., Doyle, O., \& Bliss, D. L. (2008). Reducing barriers to interprofessional training: Promoting interprofessional cultural competence. Journal of Interprofessional Care, 22(4), 417-428. doi: 10.1080/13561820802190442

Rosen, P. (2004). Supporting women in labor: Analysis of different types of caregivers. Journal of Midwifery and Women's Health, 49(1), 24-31. doi: 10.1016/j.jmwh.2003.10.013

Roth, L. M., Heidbreder, N., Henley, M. M., Marek, M., Naiman-Sessions, M., Torres, J. M., \& Morton, C. H. (2014). Maternity support survey: A report on the cross-national survey of doulas, childbirth educators and labor and delivery nurses in the United States and Canada. Retrieved from http: http://www.maternitysupportsurvey.com/Maternity_Care_Survey/HOME.html

Sleutel, M., Schultz, S., \& Wyble, K. (2007). Nurses' views of factors that help and hinder their intrapartum care. Journal of Obstetric, Gynecologic and Neonatal Nursing, 36(3), 203211. doi: 10.1111/j.1552-6909.2007.00146.x

Sosa, R., Kennell, J. H., Robertson, S., \& Urrutia, J. (1980). The effect of a supportive companion on perinatal problems, length of labor and mother-infant interaction. New England Journal of Medicine, 303, 597-600.

Statistics Canada. (2013). Table 102-4516 - Live births and fetal deaths (stillbirths), by place of birth (hospital and non-hospital), Canada, provinces and territories. Retrieved from CANSIM website: http://www5.statcan.gc.ca/cansim/ (searchable by table number). 
Stevens, J., Dahlen, H., Peters, K., \& Jackson, D. (2011). Midwives’ and doulas’ perspectives of the role of the doula in Australia: A qualitative study. Midwifery, 27, 509-516.

Tajfel, H. (1981). Human groups and social categories: Studies in social psychology. Cambridge UK: Cambridge University Press.

Torres, J. M. (2013). Breast milk and labour support: Lactation consultants' and doulas' strategies for navigating the medical context of maternity care. Sociology of Health \& Illness, 35(6), 924-938. doi: 10.1111/1467-9566.12010

Torres, J. M. C. (2015). Families, markets, and medicalization: The role of paid support for childbirth and breastfeeding. Qualitative Health Research, 25(7), 899-911. doi: $10.1177 / 1049732314553991$

Tumblin, A., \& Simkin, P. (2001). Pregnant women's perceptions of their nurses rolen during labor and delivery. Birth, 28, 52-56.

Zajonc, R. B. (1968). Attitudinal effects of mere exposure. Journal of Personality and Social Psychology, 9(2), 1-27. 